\title{
Estimation for the Number of Sound Sources Using Directional Histogram of Observed Mixture Signals
}

\author{
Yuta Kawakami $^{\mathrm{a}, *}$, Takaaki Ishibashi ${ }^{\mathrm{a}}$ \\ ${ }^{a}$ Department of Information, Communication and Electronic Engineering, \\ National Institute of Technology, Kumamoto College \\ 2659-2 Suya, Koshi, Kumamoto 861-1102, Japan \\ *Corresponding Author: te14kawakami@g.kumamoto-nct.ac.jp
}

\begin{abstract}
This paper proposes a number estimation method for sound source signals without information of sources and transfer functions. The proposed method can estimate the number of sound sources using only observed mixture signals with two microphones. A joint distribution of mixture signals has the same number of linear components as the number of sound sources. Therefore, we can estimate the source number using directional histogram of mixture signals. Furthermore, in order to be robust against outliers, the proposed method removes very small amplitude components without speech. The method works well from several simulations in the case of simultaneous utterances of multiple speakers.
\end{abstract}

Keywords: source number estimation, directional histogram, two-channel microphone system.

\section{Introduction}

The speech recognition technology has significantly been improved to achieve provision of speech recognition engine with extremely high recognition capabilities for the case of ideal environments, i.e. no surrounding noise. However, it is still difficult to attain a desirable recognition rate in a household or office where there are daily activities noises. Therefore, a certain preprocessing before recognition is needed to reduce the noises and to select the target speech signal.

Many noise reduction methods such as ICA (Independent Component Analysis) ${ }^{(1,2,3)}$, SS (Spectral Subtraction) ${ }^{(4)}$, SAFIA (sound source Segregation based on estimating incident Angle of each Frequency component of Input signals Acquired by multiple microphones) ${ }^{(5)}$ and NMF (Nonnegative Matrix Factorization) ${ }^{(6)}$ have been proposed. The authors have already proposed some estimating method ${ }^{(7,8,9)}$ for the target source signals.

The original source signals can be recovered using these estimation methods in the condition which the number of the source signals $N$ is known. However, the separation performance of the methods often deteriorates when $N$ is unknown. There has been proposed an estimation method for the sources number ${ }^{(10)}$. It functions well if the number of the sources is equal to or less than that of the sensors but it fails if the former is larger than the latter.

In order to estimate the original source signals using these methods, it is important that these estimation methods know the number of the source signals. In this paper, we propose an estimation method for the number of the source signals. The proposed method can estimate the number of sound sources using only observed mixture signals with two microphones. A joint distribution of mixture signals has the same number of linear components as the number of sound sources. Therefore, we can estimate the number of the peaks in the joint distribution for the source number using directional histogram of mixture signals. Furthermore, in order to be robust against outliers, the proposed method removes very small amplitude components without speech.

\section{Features of Observed Mixture Signals}

\subsection{Observed Mixture Signals}

When some sound sources $s_{n}(t)(n=1,2, \cdots, N)$ are observed with some microphones, the observed mixture signals $x_{m}(t)(m=1,2, \cdots, M)$ are expressed as follows.

$$
x_{m}(t)=\sum_{n=1}^{N} a_{m n} s_{n}(t)
$$




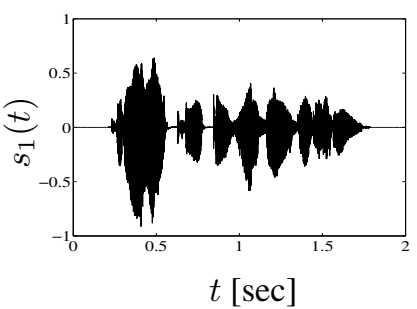

(a) Source signal $s_{1}(t)$

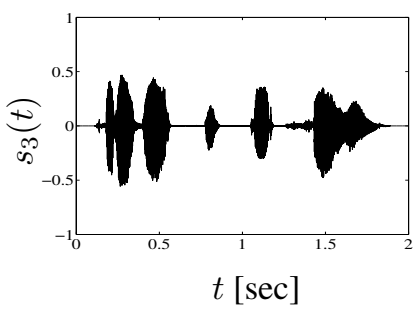

(c) Source signal $s_{3}(t)$

Fig. 1: Source signals.

where $a_{m n}$ denotes a mixing parameter, $t$ denotes index of time series, $N$ and $M$ are the number of sound sources and mixture signals, respectively.

In a real environment, the signals observed at microphones are not instantaneous mixtures but are convoluted version of the sound sources as

$$
x_{m}(t)=\sum_{n=1}^{N} \sum_{\tau} a_{m n}(\tau) s_{n}(t-\tau)
$$

where $a_{m n}(\tau)$ denotes transfer functions from the $n$-th sources to the $m$-th microphones. The mixtures $x_{m}(t)$ are transformed into the short time spectra by the discrete Fourier transform to process in frequency domain as follows.

$$
x_{m}(\omega, k)=\sum_{t} e^{-j \omega t} x_{m}(t) w(t-k \tau)
$$

where $\omega$ denotes a frequency, $k$ the frame number, $\tau$ the frame shift time and $w(t)$ a window function. In the frequency domain, the mixtures are approximated as follows.

$$
\boldsymbol{x}(\omega, k)=\sum_{n=1}^{N} a_{m n}(\omega) s_{n}(\omega, k)
$$

By expressing as Eq (4), the convolution mixing process can be thought of as an instantaneous mixing process. On account of this, there have been reported many trials to separate the convoluted mixtures in the frequency domain. In the following, we discuss in the instantaneous mixture model for simplicity.

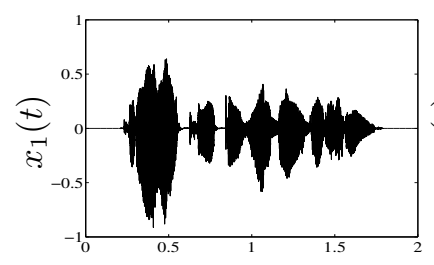

$t[\mathrm{sec}]$

(a) Observed signal $x_{1}(t)$

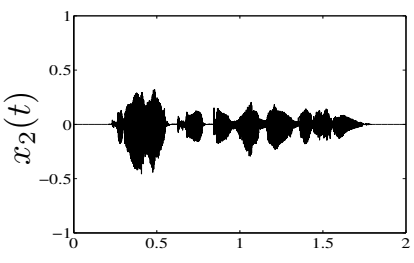

$t$ [sec]

(b) Observed signal $x_{2}(t)$
Fig. 2: Observed signals in the case of $N=1$.

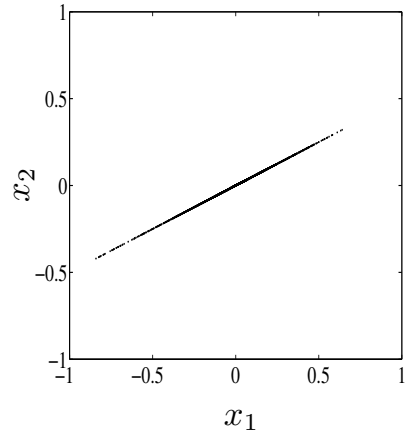

(a) Joint distribution

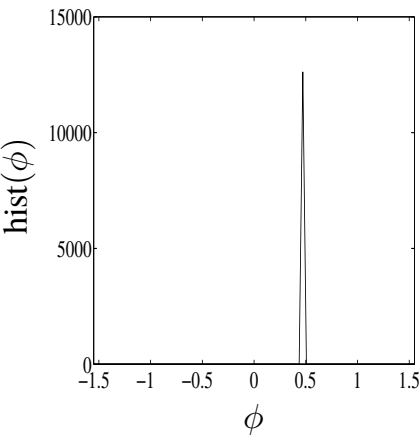

(b) Histogram
Fig. 3: Joint distribution and histogram in case of $N=1$.

\subsection{Joint Distribution and Histogram}

Consider three source signals $s_{n}(t)(n=1,2,3)$ which are human speeches shown in Fig. 1. In the following, we consider the sound signals as stochastic varieties and omit the time series $t$. If there is no active source, it is clearly that the observed signals doesn't have power. Therefore, we can estimate $N=0$ in the case which the power of the observed signals is very small.

When only $s_{1}$ is active, the waveforms $x_{1}$ and $x_{2}$ observed at the sensors are depicted as in Fig. 2. Their joint distribution is shown in Fig. 3(a) where the horizontal and the vertical axis are denoted by $x_{1}$ and $x_{2}$, respectively. Since $x_{1}$ and $x_{2}$ are completely similar, the joint distribution is expressed by a straight line. This fact implies that the distribution has onedimensional structure in the case of one active source. From the Fig. 3(a), we calculate as

$$
\phi=\tan ^{-1} \frac{x_{2}}{x_{1}}
$$

and we make the histogram hist $(\phi)$ as shown in Fig. 3 (b) using all $\phi$. The histogram of the joint distributions has only one peak as shown in Fig. 3(b). The horizontal axis denotes the direction of the ratio of the mixture signals from $-\frac{\pi}{2}$ to $\frac{\pi}{2}$ and the vertical axis denotes the frequency. 


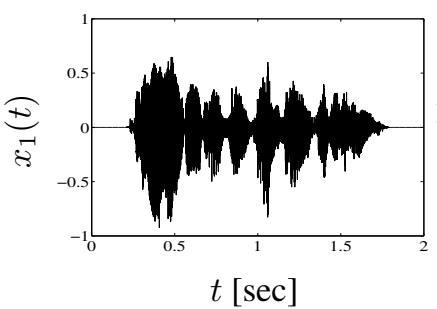

(a) Observed signal $x_{1}(t)$

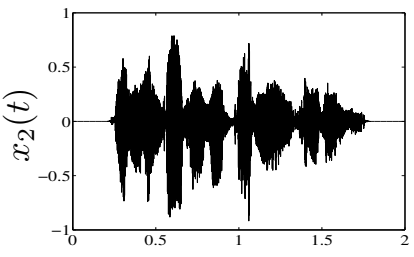

$t[\mathrm{sec}]$
Fig. 4: Observed signals in the case of $N=2$.

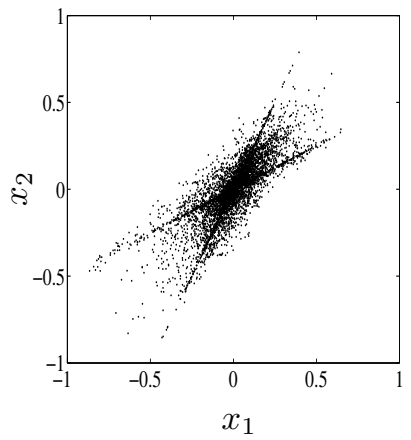

(a) joint distribution

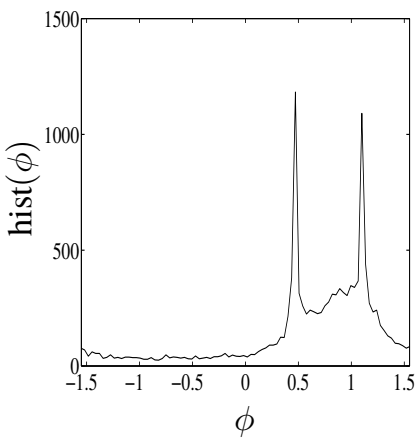

(b) histogram
Fig. 5: Joint distribution and histogram in the case of $N=2$.

In the case of two active sources, $s_{1}$ and $s_{2}$, the observed mixture signals $x_{1}$ and $x_{2}$ are shown in Fig. 4. As shown in Fig. 5(a), it is found that the joint distribution has two liner components. For clarity the linear components, directions $\phi$ of distribution are calculated as Eq (5). Fig. 5(b) shows the histogram. In the figure, two peaks are clearly seen.

In the case of three active sources, $s_{1}, s_{2}$ and $s_{3}$, the observed mixture signals $x_{1}$ and $x_{2}$ are shown in Fig. 6. Their joint distribution and the histogram are shown in Fig. 7(a) and (b), respectively. In these figure, the dense crossing lines are still discernible and three peaks are recognizable.

From these facts, the joint distribution is considered to take on a peculiar structure depending on the number of the sources. Their histograms have the same number of peaks as the sources signals. Therefore, we can estimate the number of the source signals by finding the number of the peaks.

\section{Estimation for the Number of Sources}

\subsection{Source Number Estimation}

From the above discussions, in the case which the source signals exist at the same time, the joint distribution made from the observed signals has straight lines depend on the number of sources. Then, it means that the number of source signals

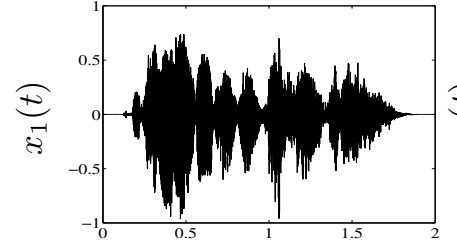

$t[\mathrm{sec}]$

(a) Observed signal $x_{1}(t)$

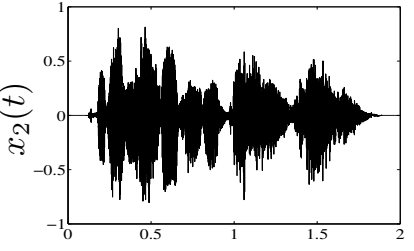

$t$ [sec]
Fig. 6: Observed signals in the case of $N=3$.

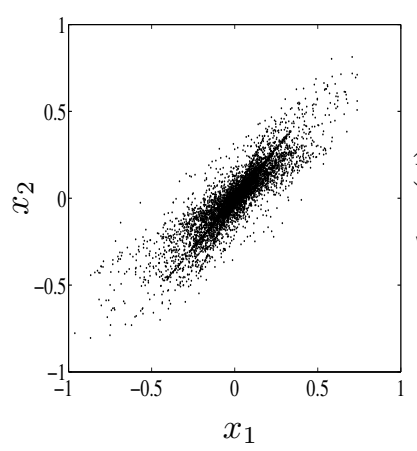

(a) joint distribution

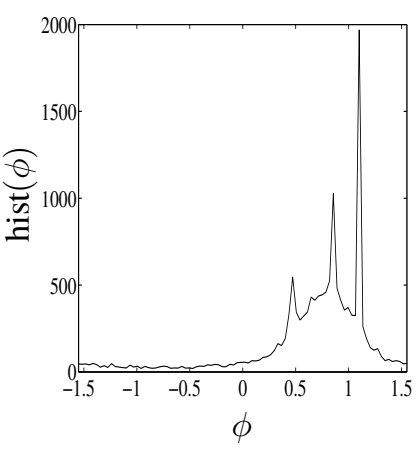

(b) histogram
Fig. 7: Joint distribution and histogram in the case of $N=3$.

can be estimated by extracting the peaks of the histogram.

We define the histogram difference $\Delta \phi_{l}$ as follows.

$$
\Delta \phi_{l}=\phi_{l}-\phi_{l-1}
$$

where $l=1,2, \cdots, L$ denotes the number of flames of the histogram. The number of sound sources can be estimated by counting the number in the case which the histogram difference $\Delta \phi_{l}$ is larger than the threshold value.

\subsection{Robust Number Estimation Method for Outliers}

As can be seen from the Figs. 5(a) and 7(a), there are many components with very small amplitudes in the distribution. When the proposed method calculates angles of these small amplitude components, unnecessary values may be generated. When the amplitude of the observed signal is very small, the ratio $\frac{x_{2}}{x_{1}}$ of the observed signals as shown in Eq (5) becomes 0 or infinity. Trigonometric functions of Eq (5) can not be calculated. Therefore, we remove components with small amplitude. By the processing, it is possible to remove many small peak components in the histogram.

\section{Simulation}

In order to verify our proposals, several simulations were carried out. The source signals $s_{1}(t) s_{2}(t)$ and $s_{3}(t)$ were 
human speech signals in the database ${ }^{(10)}$. These signals were sampled at the rate of $8[\mathrm{kHz}]$ with 16 [bit] resolution. Using these sources, the mixture signals are generated by Eq (2) with the mixing parameters $a_{m n}=0.9 \pm \eta(m=n)$ and $a_{m n}=0.6 \pm \eta(m \neq n), \eta$ denotes a random value from 0 to 0.1 .

Under this condition, Using 6 [sec] speech signals, 30 mixture signals patterns (permutation of 3 males and 3 females: ${ }_{6} \mathrm{P}_{2}$ ) were generated in the case of two source condition. In addition to it, 20 mixture signals patterns (combination of 3 males and 3 females: ${ }_{6} \mathrm{C}_{3}$ ) ware generated in the case of three source condition. The removal rate of small amplitude values was 1 percent of all data points. We confirmed that the method can estimate the number of the source signals in all data completely.

When 2 [sec] speech signal data is used, the proposed method failed the estimation in some cases. The reason is that there is no breathing or silent interval in the human speech, so the peak does not appear in the histogram of the observed mixture signals. From these results, it is found that the proposed method is effective for estimating the number of sound sources when breathing and silent interval are included in observed mixed speech.

\section{Conclusion}

This paper proposes a number estimation method for sound source signals without information of sources and transfer functions in order to estimate the source signals in blind source separation. The proposed method can estimate the number of sound sources using only observed mixture signals with two microphones. A joint distribution of two mixture signals has the same number of linear components as the number of sound sources.

Using this fact, we propose an estimation method for the source based on the directional histogram of the mixture signals. The proposed method can estimate the number of the source signals by counting the number of the peaks of the histogram. Furthermore, in order to be robust against outliers, the proposed method removes very small amplitude components without speech. From the simulation, it is found that the proposed method works well for estimating the number of sound sources when breathing and silent interval are included in observed mixed speech.

\section{Acknowledgment}

This work was supported by JSPS KAKENHI Grant Number $16 \mathrm{~K} 21583$.

\section{References}

(1) A. Hyvärinen, J. Karhunen, and E. Oja: "Independent component analysis", John Wiley \& Sons, Ltd, 1998

(2) A. Cichocki and S. Amari: "Adaptive blind signal and image processing, learning algorithm and applications", John Wiley \& Sons, Ltd, 2002

(3) S. Makino, T.-W. Lee and H. Sawada: "Blind speech separation”, Springer, 2007

(4) S. F. Boll: "Suppression of acoustic noise in speech using spectral subtraction", IEEE Transactions on Acoustics, Speech and Signal Processing, Vol. ASSP-27, No. 2, pp. 113-120, 1979

(5) M. Aoki, M. Okamoto, S. Aoki, H. Matsui, T. Sakurai and Y. Kaneda: "Sound source segregation based on estimating incident angle of each frequency component of input signals acquired by multiple microphones, Acoustical Science and Technology", Vol. 22, No. 2, pp. 149-157, 2001

(6) A. Cichocki, R. Zdunek and S. Amari: "Nonnegative matrix and tensor factorization", IEEE Signal Processing Magazine, Vol. 25, No. 1, pp. 142-145, 2008

(7) T. Ishibashi, K. Higuchi, C. Okuma and K. Hayama: "Noise reduction method for multiple sound source signals and its application to two-channel microphone system," Proceedings of the 5th IIAE International Conference on Industrial Application Engineering 2017, pp. 336-339, 2017

(8) K. Higuchi, C. Okuma and T. Ishibashi: “ Blind source separation and human speech extraction for three sound sources using silent interval," The 5th IIAE International Conference on Intelligent Systems and Image Processing 2017, pp. 205-208, 2017

(9) T. Ishibashi, K. Inoue, C. Okuma and K. Eguchi: “ Low distortion target speech extraction method using twochannel microphone system," The 6th IIAE International Conference on Intelligent Systems and Image Processing 2018, pp. 428-431, 2018

(10) H. Sawada, R. Mukai, S. Araki, S. Makino: “ Estimating the number of sources using independent component analysis," Acoustical Science and Technology, the Acoustical Society of Japan, Vol. 26, No. 5, pp. 450-452, 2005

(11) Acoustical Society of Japan: "ASJ continuous speech corpus Japanese newspaper article sentences", JNAS Vols. 1-16, 1997 